\title{
Environmental impact on activity level and fecal glucocorticoid metabolite concentration of African elephants and black rhinoceros at brookfield zoo
}

\begin{abstract}
Visitors and the accompanying increased sound levels are two of the factors of daily life for many zoo-housed animals. Here the impact different seasons have on the physiological and behavioral responses of two African elephants, Loxodonta africanus, and three Black rhinoceros, Diceros bicornis, were evaluated at the Brookfield Zoo. All study animals showed the highest fecal glucocorticoid metabolite (FGM) concentrations during the summer when exhibit attendance is the highest, and lowest FGM concentrations in the winter when exhibit attendance is the lowest. Interestingly, black rhinoceros were less active when FGM levels and exhibit attendance were higher and elephants more active. Unexpectedly, ambient sound levels followed no clear pattern between seasons. Friedman's tests showed FGM concentrations and exhibit attendance was significantly higher in the summer than in winter. The results highlight that exhibit attendance may be associated with some changes in adrenal and behavioral responses of zoo animals, but there is also substantial individual variation within and across species in their responses to the same environmental factors.
\end{abstract}

Keywords: glucocorticoids, welfare, elephant, rhinoceros, environmental impact
Volume 3 Issue 2 - 2018

\author{
Jocelyn L Bryant,' Nadja CWielebnowski ${ }^{2}$ \\ 'Chicago Zoological Society, USA \\ 2Oregon Zoo, USA
}

Correspondence: Jocelyn Bryant, Department of Animal Welfare Research, Brookfield Zoo, 3300 Golf Rd, Brookfield, IL 605।3, USA, Email Jocelyn.Bryant@czs.org

Received: February 20, 2018| Published: March 13, 2018

\section{Introduction}

So far few studies have investigated the impact of season, associated exhibit attendance and ambient sound on zoo animals in general, yet there is increasing public concern that some of these factors may negatively impact zoo animals. A study on giant pandas at the San Diego Zoo Owen at el. ${ }^{1}$ investigated the impact of sound on adrenal and behavioral indicators of stress and found that louder sound was associated with an increase of stress-related behaviors, such as vocalization, door scratching, increased locomotion, and/or increased urinary glucocorticoid levels in all study animals. Carlstead at al. ${ }^{2}$ found higher fecal glucocorticoid levels (FGM) in black rhinoceros housed in enclosures with a greater portion of the perimeter accessible to visitors. Two other studies, one on orangutans at Chester Zoo, UK, Birke et al. ${ }^{3}$ and the other on black rhinoceros at various US zoos Carlstead ${ }^{4,5}$ also documented an increase in some behaviors associated with higher levels of stress when larger groups of people were present and/or sound levels were higher. Black rhinoceros showed an increase in fear-related behavior traits such as shyness and anxiety. Unfortunately, the two latter studies were not able to assess physiological indicators in addition to behavioral assessments. Combining more than one potential indicator when looking for increased stress responses is key to better understanding the impact of various factors on an individual's welfare Wielebnowski. ${ }^{6}$ The growing availability of reliable non-invasive measurement techniques, such as FGM monitoring, makes longitudinal studies using physiological indicators in combination with behavioral observations increasingly feasible Möstl \& Palme, ${ }^{7}$ Millspaugh \& Washburn. ${ }^{8}$

Zoo animals regularly experience a variety of sound levels across the seasons due to weather conditions, public zoo events, construction, etc. The cumulative sound measured at two Northern California zoos including visitor activity, maintenance equipment, airplanes, and water features averaged 70 decibels (dB) Morgan \& Tromborg. ${ }^{9}$ in a recent review paper, compared these decibel levels to those measured in some of the original natural habitats of species commonly housed in zoos. They discovered that sound levels in zoos were usually two to three times higher than the decibel levels found in natural environments. Recorded sound was as low as $20 \mathrm{~dB}$ in savannah habitats and as high as $40 \mathrm{~dB}$ in rain forests. It is very likely that most zoo animals will experience much higher levels of sound than their wild counterparts. Yet whether this difference in ambient sound levels has any significant impact on their behavior and physiological state remains unknown. Studies conducted on laboratory and farm animals may provide some indication about possible effects. For instance, laboratory rodents expressed symptoms of increased physiological stress as ambient sound approached $85 \mathrm{~dB}$ for several hours Anthony \& Ackerman. ${ }^{10}$ Furthermore, in some laboratory species, sources of loud sounds such as construction, machinery, and sirens appeared to have detrimental effects, most seriously seizures, hearing loss, and increased aggression Gamble, ${ }^{11}$ Patterson Kane \& Farnworth. ${ }^{12}$ In particular, rats exposed to various sounds during pregnancy and copulation showed a decline in birth rates Sato et al. ${ }^{13}$ While some level of stress and associated stress responses represent a normal part of animals' lives, repeated acute stress and continuous chronic stress can have long-term damaging effects such as decreased immune function, decreased reproductive success, and metabolic disruption Turneret et al. ${ }^{14}$ In wild animals seasonal environmental stressors that may negatively affect their well-being may include increased human activity as well as high levels of sound Teixeira et al. ${ }^{15}$

In this study we measured one aspect of adrenal activity using FGM monitoring and activity level employing direct quantitative observations in two charismatic pachyderm species commonly 
housed in zoos worldwide. We then examined how seasons and some commonly experienced environmental factors, such as exhibit attendance and ambient sound levels, could be associated with changes in physiological and behavioral measures. The outcomes of this study will provide a first indication about possible seasonal impacts of these variables on some aspects of individual animals' welfare and hopefully provide incentive for further research on this topic. We hypothesize:

a. An increase in FGM concentrations in pachyderms is associated with increased visitor numbers at the exhibit.

b. An increase in FGM concentrations in pachyderms is associated with higher noise levels at the exhibit.

c. An increase in activity levels in pachyderms is associated with increased noise level and visitor numbers at the exhibit.

Ultimately studies like this may help to inform future exhibit design and improvements in housing conditions that would help to alleviate potential negative impacts of environmental variables throughout the seasons.

\section{Methods}

\section{Study animals}

Two female African elephants (Loxodonta africana), and one female and two male black rhinoceros (Diceros bicornis), constituting all animals of these two species at Brookfield Zoo at the time of the study were part of this project. All the individuals were housed in the pachyderm building at the zoo (Table 1). Study animals were in good physical health aside from African elephant E2 who had a congenital kidney issue but showed no symptoms of clinical illness. Also, African elephant E1 experienced recurring foot problems, but was otherwise in excellent health.

Table I Study animal information: ID, gender, age, and dates of data collection for each study animal

\begin{tabular}{llllll}
\hline Project ID (name) & Species & Wild vs Captive Born & Age & Years at BZ & Data Collection Span \\
\hline EI (Affie) & African Elephant & Wild & 39 & 30 & $3 / 9 / 07-5 / 14 / 08$ \\
E2 (Christy) & African Elephant & Wild & 27 & 25 & $3 / 9 / 07-5 / 14 / 08$ \\
RI (Kabisa) & Black Rhino & Captive & 23 & 23 & $3 / 5 / 07-5 / 14 / 08$ \\
R2 (Nakili) & Black Rhino & Captive & 16 & 15 & $7 / 30 / 07-5 / 14 / 08$ \\
R3 (Shima) & Black Rhino & Captive & 23 & 23 & $3 / 5 / 07-5 / 14 / 08$ \\
\hline
\end{tabular}

The pachyderm building is an open access building, which means that the public can view the animals in both their outdoor and indoor enclosures at all times. The inside enclosures are maintained at 21 ${ }^{\circ} \mathrm{C}$ in the winter and there is no air conditioning, only air circulation in the summer, which can produce temperatures over $30^{\circ} \mathrm{C}$. The light cycle is natural as there are skylights. The elephants and black rhinoceros remain inside from November through March unless outside temperature reaches near $10{ }^{\circ} \mathrm{C}$. The rest of the year they are allowed outside if the temperature is over $10^{\circ} \mathrm{C}$ and no thunderstorms are predicted. All study animals are exhibited only outside on good weather days. The outside yard for the elephants is 3,429 sq meters and their 3 inside holding stalls are 663 sq meters each with a 9 meter high ceiling. The inside stalls have rubberized flooring, scratching posts, rocky walls, fresh water drinkers, hanging feeders and chains from which to hang behavioral enrichment, and the outside yard contains a fake feeder tree, shade, pool, a sandy/clay substrate and are entirely visible to the public. The 2 black rhinoceros on the south side of the pachyderm building, R3 and R2, are always separated and share 3 inside stalls, 363sq meters each. They are given access to either 1 or 2 inside stalls on alternating days year-round giving each rhinoceros more interior space every other day. The inside stalls have concrete floors, scratching posts, rocky walls, fresh water drinkers, hanging feeders, and chains to hang behavioral enrichment, and the yards contain pools, mud wallows, trees, a sandy dirt substrate and are completely visible to the public. On good weather days, R3 has access to a 2,057sq meter yard and R2 has access to a 3,703sq meter yard. R1 is housed on the north side of the building and has access to two inside stalls, 236sq meters total, and a separate 4,481sq meter outside yard. Feeding schedules and food items are very similar for both, elephants and rhinoceros; mixed hay (Bermuda grass, timothy hay, and alfalfa) is provided in the morning and afternoon with various produce and bread. Water is available ad libitum.

\section{Behavioral observations}

We performed behavioral observations using simple check sheets and direct observations. We conducted three daily (Monday-Friday) 5 minute observations per animal with one minute instantaneous sampling. The study was conducted from March 2007-May 2008, resulting in a total of approximately 81 hours of behavioral data per animal. Observations occurred at approximately 11:30, 13:30, and 15:30 each day. Two researchers performed most observations and two volunteers covered time slots when needed if possible. To keep behavioral data collection simple and reliable across observers we focused on recording only observable behavioral states that would be representative of activity level. Inactive behavior would be lie or stand, and active behavior would be locomotion, grooming/maintenance, feeding, play and social interactions.

\section{Data collection of environmental variables}

Brookfield Zoo is open to the public daily year-round. We monitored exhibit attendance using counts during each observation. For ease of estimation we divided visitor counts into the following categories: none, 1-5, 6-10,11-15, and greater than 16 people. At the end of every minute during each five minute observation period, we estimated the number of visitors standing in front of the exhibit and checked the appropriate category for each count. Visitors walking along the pathways behind those stopped at the exhibit were not included in the estimate. 
We obtained temperature data from a weather station located on Brookfield Zoo's property and expressed values as average daily temperature. We used sound meters to measure ambient sound levels in decibels in front of each animal exhibit during every observation. Specifically, we utilized a hand-held sound meter with a 30-130 dB range (Sper Scientific, Mini Sound Meter, model \# 850014) during each behavioral observation and placed on top of fencing or railing in front of the exhibit next to the observer.

\section{Fecal hormone metabolite analysis}

Zookeepers collected fecal samples three times per week (approximately 195 samples per study animal), evenly distributing collection dates, for the entire study period. After collection, keepers froze samples immediately in a freezer at $-20^{\circ}$ Celsius. Fecal glucocorticoid metabolites (FGM) were extracted using $80 \%$ ethanol in distilled $\mathrm{H}_{2} \mathrm{O}$. First, we weighed $0.5 \mathrm{~g}(+/-0.05 \mathrm{~g})$ of each fecal sample into $16 \times 125 \mathrm{~mm}$ polypropylene tubes (Mettler balance, model \#AB104-5). Then, added $5 \mathrm{~mL}$ of $80 \%$ ethanol solution to each extraction tube. Each tube was vortexed and placed on a rotator (Labline Maxi Rotator, model \#4631) overnight for 14-18 hrs. We then centrifuged the tubes for $15 \mathrm{~min}$ at $1500 \mathrm{rpm}$ (Marathon 3000R centrifuge, model \#120). For each sample, we pipetted $1 \mathrm{~mL}$ of supernatant into $1 \mathrm{~mL}$ of assay buffer $(0.1 \mathrm{M}$ phosphate buffered saline containing $1 \% \mathrm{BSA}, \mathrm{pH} 7.0$ ) and into $12 \times 75 \mathrm{~mm}$ polypropylene tubes. Lastly, we capped each extract tightly to avoid evaporation and stored frozen at $-20^{\circ}$ Celsius until assay analyses.

We assayed black rhinoceros samples using a previously established and validated in-house corticosterone EIA assay. However, we did not use this in-house assay for African elephants. Because each species excretes a different array of hormone metabolites in the feces it is not uncommon that assays that work for one species will not be applicable for another (REF REQUEST). Elephant samples were instead assayed using a commercially available corticosterone EIA kit (Enzo Life Sciences, Ann Arbor, MI, USA), to determine FGM concentrations. We validated each assay biochemically and biologically for each species. Biochemical validation of the assays consisted of the following: 1. parallelism with the standard curve, and 2. recovery to determine the percentage of exogenous hormone measured. For the recovery test one sample was spiked with the highest five standards. For the biological validation we monitored physiological response to a known stressor, such as a vet exam, transport to another facility, or major social change. More specifically, for black rhinoceros, we monitored the adrenal activity of a female being transported to another zoological institution. Several fecal samples were collected prior, during, and a couple weeks after the transport. For African elephants we monitored a major social change that occurred following the study period. A female elephant housed alone showed associated indications of behavioral and physiological stress. We collected fecal samples continuously throughout the herd changes and we measured adrenal activity before and after this event (Figure 1) (Figure 2).

For the in-house corticosterone assay, the anti-body was diluted 1:7,500 and the working HRP dilution was 1:25,000. C. Munro (University of California-Davis, Davis, CA) prepared and supplied the in-house corticosterone anti-body (CJM006) and conjugate used for the rhinoceros samples. The standard curve range was $0.09 \mathrm{ng} /$ $\mathrm{mL}$ to $25 \mathrm{ng} / \mathrm{mL}$ and the sample volume was $100 \mathrm{uL}$ per well. Cross- reactivity of the CJM006 corticosterone anti-body is as follows: $100 \%$ corticosterone, $14.25 \%$ desoxycorticosterone, $2.65 \%$ progesterone, $0.90 \%$ tetrahydrocorticosterone, $0.64 \%$ testosterone, $0.07 \%$ prednisolone, and any other steroids were $<0.01 \%$. Assay sensitivity was $0.09 \mathrm{ng} / \mathrm{mL}$ and the intra-assay coefficient of variation was $7.14 \%$ at $35.02 \%$ binding. Inter-assay variation was $12.77 \%$ at $31.88 \%$ binding and $16.14 \%$ at $66.67 \%$ binding. The recovery of exogenous corticosterone $(1.56-25 \mathrm{ng} / \mathrm{mL})$ was $76.84 \%$ in black rhinoceros. Enzo Life Sciences supplied the corticosterone commercial EIA kit used to analyze elephant samples (Ann Arbor, MI, USA, catalog \# 901-097). The manufacturer supplied all needed instructions and components. We read plates on a photospectrometer plate reader (Dynex MRX Revelation) at $405 \mathrm{~nm}$. The cross-reactivity of the Enzo Life Sciences corticosterone anti-body was as follows: $100 \%$ corticosterone, $28.6 \%$ desoxycorticosterone, $1.7 \%$ progesterone, $0.28 \%$ tetrahydrocorticosterone, $0.18 \%$ aldosterone, $0.13 \%$ testosterone and any other steroids were $<0.05 \%$. Assay sensitivity was $26.99 \mathrm{pg} / \mathrm{mL}$ and the intra-assay coefficient of variation was $12.34 \%$ at $53.38 \%$ binding. Inter-assay variation was $10.38 \%$ at $53.66 \%$ binding and $15.89 \%$ at $68.46 \%$ binding. The recovery of exogenous corticosterone $(250-4000 \mathrm{pg} / \mathrm{mL})$ was $100 \%$ in African elephant. We expressed all hormone data in $\mathrm{ng} / \mathrm{g}$ wet feces.

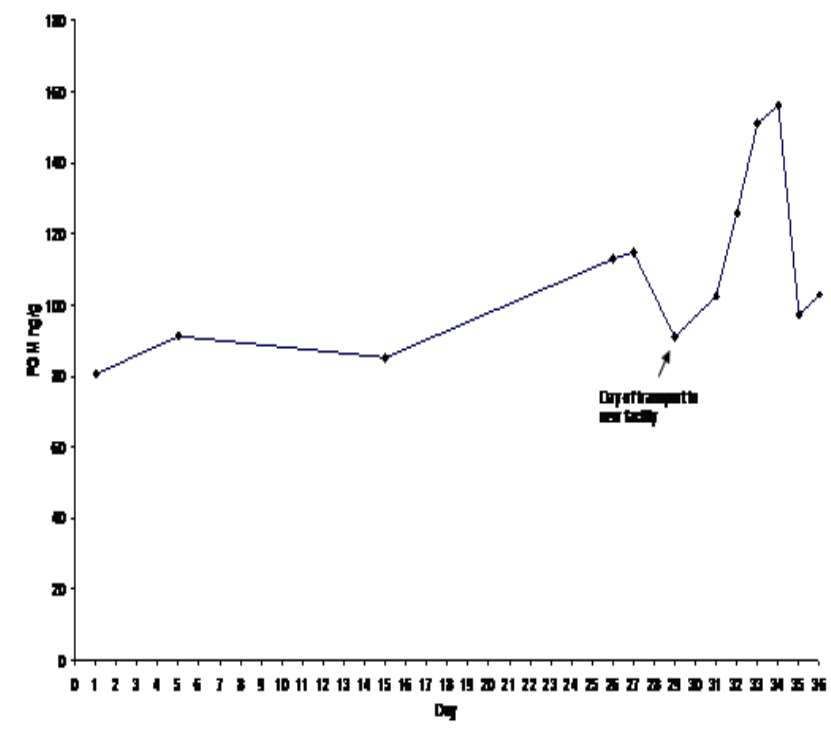

Figure I Black rhinoceros biological validation monitoring fecal hormone metabolite (FGM) concentrations before, during, and after transport to a new facility. Transport occurred on Day $29(6 / 16 / 08)$ and levels began to increase on Day 31.

\section{Data analysis}

All data variables for each individual were compiled as seasonal averages. These variables included FGM concentrations, proportion of time active, visitor numbers, and sound level $(\mathrm{dB})$. Seasons were defined as winter: December, January, and February; spring: March, April, and May; summer: June, July, and August; and fall: September, October, and November. The behavioral state "active" includes locomotion, feeding, grooming, manipulating objects or their environment and social interactions. Once averages of the four 
variables were obtained for each of the four seasons, all data sets were tested for normal distribution. Not all data was normally distributed, therefore we used non-parametric tests. First a Friedman's test was run on the four variables to test for significance across seasons. Then, all pair wise comparisons were performed on any variables showing significant results from the Friedman's tests. We took a more conservative approach using all pair wise comparisons versus Wilcoxon signed rank tests, to ensure accurate significant results, Cody \& Smith. ${ }^{16}$ All statistical analyses were performed using SPSS version 22 and we applied a $P \leq 0.05$ significance level for all tests.

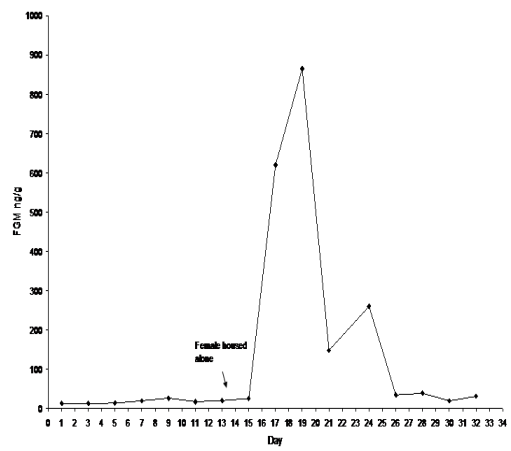

Figure 2African elephant biological validation monitoring FGM concentrations before, during and after a major change in social housing. Within 48 hours of the change FGM levels became elevated and returned to baseline 10 days later.

Table 2 The means of FGM concentration, proportion of time active, exhibit attendance, and ambient sound levels for each individual across all seasons

\begin{tabular}{|c|c|c|c|c|}
\hline & FGM ng/g $( \pm$ SD $)$ & Proportion Time Active & Exhibit Attendance & Ambient Sound (dB) \\
\hline \multicolumn{5}{|l|}{ E1 } \\
\hline Winter & $19.41( \pm 14.05)$ & 0.29 & 1.5 & 59.44 \\
\hline Spring & $54.87( \pm 41.65)$ & 0.39 & 9.7 & 47.79 \\
\hline Summer & $87.33( \pm 63.13)$ & 0.54 & 15.29 & 72.45 \\
\hline Fall & $65.08( \pm 65.53)$ & 0.46 & 8.12 & 88.91 \\
\hline \multicolumn{5}{|l|}{ E2 } \\
\hline Winter & $19.00( \pm 14.14)$ & 0.28 & 1.41 & 62.83 \\
\hline Spring & $47.88( \pm 41.76)$ & 0.46 & 9.7 & 47.77 \\
\hline Summer & $61.00( \pm 22.73)$ & 0.57 & 15.38 & 61.49 \\
\hline Fall & $49.00( \pm 24.89)$ & 0.36 & 8.09 & 76.84 \\
\hline \multicolumn{5}{|l|}{ R1 } \\
\hline Winter & $196.51( \pm 45.45)$ & 0.58 & 0.64 & 61.3 \\
\hline Spring & $218.22( \pm 100.82)$ & 0.48 & 3.41 & 54.83 \\
\hline Summer & $288.80( \pm 58.58)$ & 0.42 & 4.05 & 65.74 \\
\hline Fall & $220.48( \pm 95.46)$ & 0.49 & 1.78 & 63.25 \\
\hline \multicolumn{5}{|l|}{$\mathbf{R 2}$} \\
\hline Winter & $209.27( \pm 43.47)$ & 0.81 & 0.54 & 60.62 \\
\hline Spring & $251.85 \pm(104.04)$ & 0.69 & 4.14 & 62.29 \\
\hline Summer & $291.40( \pm 32.19)$ & 0.57 & 5.05 & 63.26 \\
\hline Fall & $257.84( \pm 49.90)$ & 0.81 & 2.23 & 59 \\
\hline \multicolumn{5}{|l|}{$\mathbf{R 3}$} \\
\hline Winter & $77.41( \pm 103.96)$ & 0.78 & 0.6 & 60.03 \\
\hline Spring & $82.84( \pm 81.66)$ & 0.63 & 3.23 & 49.48 \\
\hline Summer & $181.41( \pm 45.73)$ & 0.51 & 5.23 & 63.58 \\
\hline Fall & $157.00( \pm 34.78)$ & 0.8 & 1.91 & 60.62 \\
\hline
\end{tabular}

Citation: Bryant JL,Wielebnowski NC. Environmental impact on activity level and fecal glucocorticoid metabolite concentration of African elephants and black rhinoceros at brookfield zoo. Int J Avian \& Wildlife Biol. 2018;3(2):94-I00. DOI: I0.I5406/ijawb.20I8.03.00064 
Table 3 Comparison of season and environmental variables of African elephant and black rhinoceros exhibits at Brookfield Zoo. Significant P values are shaded in gray

\begin{tabular}{llllll}
\hline & Winter & Spring & Summer & Fall & P values \\
\hline FGM (ng/g) & 104.32 & 131.13 & 181.99 & 149.88 & 0.002 \\
Activity Level & 0.548 & 0.53 & 0.4194 & 0.584 & 0.204 \\
Exhibit Attendance & 0.938 & 6.036 & 8.818 & 4.426 & 0.002 \\
Ambient Noise (dB) & 60.844 & 52.432 & 65.304 & 69.724 & 0.069 \\
\hline
\end{tabular}

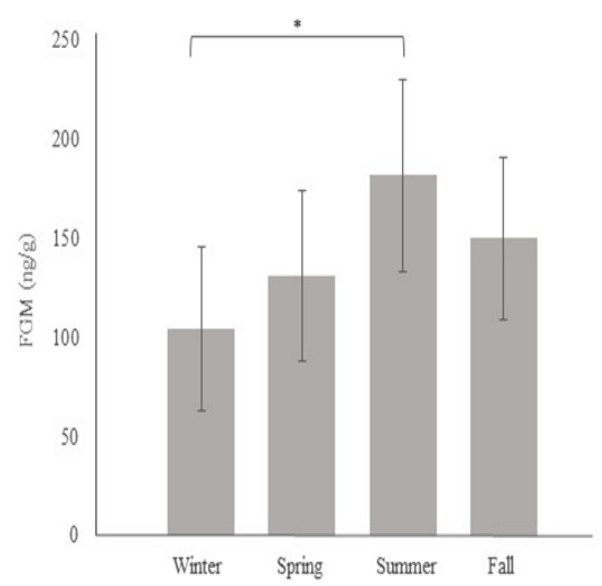

Figure 3 Average fecal glucocorticoid metabolite (FGM) concentrations of African elephants and black rhinoceros combined over seasons. Significance was found between winter and summer $(p=0.001)$ with summer having highest FGM concentrations and winter the lowest.

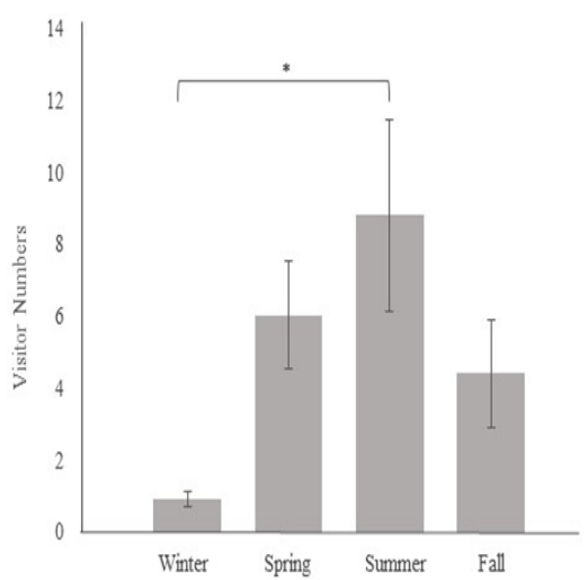

Figure 4 Average exhibit attendance for all study animals over seasons. Significance was found between winter and summer $(p=0.002)$ with summer having highest amount of visitors and winter the least.

\section{Discussion}

The primary goal of our study was to examine the seasonal impact on one aspect of adrenal activity and behavioral activity level of two highly charismatic pachyderm species, African elephants and black rhinoceros. Additionally, relationships between seasons and common environmental factors regularly experienced by zoo animals were examined. In particular, we were interested to test potential differences between seasons at a Northern location, such as Brookfield, Illinois, with relatively long cold winters, associated with low exhibit attendance and animals housed indoors for prolonged periods of time. The results of this research yielded several interesting outcomes with regard to exhibit attendance and sound and it also highlights possible differences between species and individuals in response to changing seasons.

We found increased FGM concentrations for all study animals during the summer as well as the highest exhibit attendance in the summer. Summer is the busiest time of year for the Zoo and interestingly also coincides with the time when all of the animals have consistent access to the outside yards. One factor untested was temperature, but it was assumed that there is a very strong correlation between temperature and exhibit attendance and it is therefore hard to tease apart the relative effects of each. Yet one has to keep in mind that the indoor temperature in the pachyderm house generally remains at a steady $21^{\circ} \mathrm{C}$ unless it is a very hot summer day, and African elephants and black rhinoceros do not have access to outside areas unless the air temperature is above $10^{\circ} \mathrm{C}$. Therefore outside air temperature overall may have less of an effect on the animals than on exhibit attendance, though it is important to note that sudden changes in temperature can cause a stress response Wingfield. ${ }^{17}$

In terms of visitor access to the pachyderm building, visitors can see the animals indoors and outdoors at all times during regular Zoo visitor hours. However, the Zoo is open for longer hours, nine hours, during the 'high' season (Memorial Day through Labor Day) and seven hours during the 'low' season. Based on a review of current literature Hosey ${ }^{18}$ came to the conclusion that visitor presence can result in increased stress for many zoo animals depending on the surrounding environment and exhibit. For example, increased public access around the enclosure correlates with higher FGM in black and white rhinoceros Carlstead \& Brown. ${ }^{19}$ Similarly it has been found that for some cat species, such as clouded leopards and jaguars, glucocorticoid levels were lower when animals were housed offexhibit Wielebnowski et al. ${ }^{20}$ or had ${ }^{21}$ access to more hiding spaces within their enclosure. While visitors are of course a key element of zoos, enclosure design that allows animals to retreat from 'open view' may help zoo animals cope with any real or perceived challenges in their environment. At the same time, it is also important to consider increased exercise may increase FGM as well. We also found some activity level differences related to season in the majority of our study animals. Black rhinoceros were more inactive during the summer which coincides with highest exhibit attendance and also would coincide with highest yearly temperatures. In a study on orangutans at one zoological facility, it has been noted that increased groups of visitors elicit more hiding behaviors, ${ }^{3}$ yet in many studied primates, increased human presence may also lead to more audiencedirected active behavior and/or within species aggression Mitchell, ${ }^{22}$ Lambeth, ${ }^{23}$ Hosey. ${ }^{24}$ Indeed the African elephants in this study 
showed increased activity in the summer which may account for increased levels of FGM. However, the elephant yard, as opposed to the rhinoceros yards, allows access to mud puddles, a pool, and misters which also may have helped to increase the activity level. It is generally reported though in the literature that larger groups of people may cause some atypical species behavior patterns including higher levels of inactivity). Carlstead et al. ${ }^{4,5}$ found that black rhinoceros showed more "fearful" behavior such as being timid, depressed, inactive, and less social when there was a larger amount of public access provided to the exhibit circumference. Some other species are known to regularly retreat or hide in the presence of large groups of people Kyngdon \& Stafford, ${ }^{25}$ Nogueira, Bernadi \& Nogueira. ${ }^{26}$ Based on these results we speculate that increased exhibit attendance may indeed have affected some of the changes in activity level we observed in rhinoceros and elephants during this study.

Ambient sound levels can also be a major factor affecting animal behavior and physiology. It has been shown that high levels of ambient noise can induce physiological stress responses in laboratory and zoo animals Jildge, ${ }^{27}$ Weisenberger et al. ${ }^{29}$ found sound level in natural savannah habitat to be around $20 \mathrm{~dB}$ with a typical 2-3 fold $\mathrm{dB}$ increase measured in zoos in exhibits that feature savannah habitats and associated species. Ambient sound levels during this study averaged approximately 60-65 dB throughout the year, likely associated with anthropogenic noise and a highly echoing interior exhibit. Many species, including elephants and rhinoceros, can hear sound frequencies undetectable to human ears such as infrasound for example Muggenthaler, ${ }^{29,30}$ and may therefore be more sensitive to higher noise levels. Also, several studies have found that increased noise levels can cause an increase in general activity and other behaviors that signify arousal Krebs, ${ }^{31}$ Maier, ${ }^{32}$ yet surprisingly the black rhinoceros and African elephants in our study showed sound levels did not necessarily coincide with exhibit attendance and we suspect that exhibit attendance and other environmental variables are potentially stronger factors, possibly outweighing some 'noise' effects. Overall ambient sound levels were slightly higher during spring near black rhinoceros and fall near African elephants. One reason for a difference between these species may be that all elephants were typically outdoors in good weather conditions and the elephant yard is more expansive. Visitors are usually more evenly distributed along the accessible part of the perimeter, whereas visitors are more clustered around both the outside and inside area of the rhinoceros yards. Also, there are some other methodological issues related to the sound measure locations during various times of the study that may have affected the overall outcome. There is a substantial amount of echo inside the pachyderm building and sound was often amplified inside even at relatively low visitor densities if voices were louder. It was difficult to evaluate which sound levels may have had more of a potential impact on the animals, outside without echo or inside with echo. In future studies decimeters should be used both inside and outside the exhibit areas in a variety of locations and need to be monitored continuously. Yet, we do believe, based on the relatively strong relationship observed between FGM, season, and exhibit attendance, that these factors may have outweighed the impact of sound alone.

Variation in coping mechanisms and coping styles within species and across individuals can also be a contributing factor to differences observed in FGM levels. Wingfield ${ }^{17}$ noted that animals usually establish routines within their environment with predictable changes. In addition, individual animals will develop differing routines and face different challenges depending on variation in temperament and previous experiences. Developing unique coping styles and behaviors can be a way for an animal to deal with an unpredictable or uncontrollable change in environment Weiss ${ }^{33}$ Ursin. ${ }^{34}$ Two main types of stress coping responses were distinguished by Henry \& Stephens. ${ }^{35}$

\section{i. An active response such as aggression; and}

ii. A withdrawal response such as inactivity, more commonly known as the "fight or flight" response.

It seems therefore that the observed inactivity level in black rhinoceros during times of higher visitor numbers may be a response to environmental changes, whether primarily induced by exhibit attendance, or other possibly associated factors such as temperature. The fact that elephants are more active when rhinoceros are not, or individual differences, may indicate either a difference in coping style, or it may be a reflection of the ability to become more active in a more diversified outside yard during warmer months. Although our study extended over 15 months and a detailed longitudinal amount of hormonal and behavioral data were compiled for the two species, the number of study animals was low. Based on such a small sample size it is of course difficult to draw any strong conclusions. There is a need for future research to investigate these initial findings in more detail and to specifically test potential causal relationships of the variables involved. Our data indicate however that studies investigating various aspects of visitor impact are necessary to fine-tune future exhibit design and management practices so we can accommodate both, the animals' need for retreat and privacy, as well as the visitors' expectations to not only see animals but animals that are active and show species-appropriate behaviors. ${ }^{36}$

Finally, gaining a better understanding of the causes underlying changes in stress responses and behavioral activity over time can enable animal managers and caretakers to further improve exhibit and husbandry conditions which ultimately helps to maximize the wellbeing of zoo animals.

\section{Acknowledgements}

Many thanks for the valuable contributions of Christina Pawlik, Jessica Whitham-Pacione, Lance Miller, and the volunteers who generously helped with the collection of behavioral data. A special thanks to the Brookfield Zoo's pachyderm keepers: George Morgan, Scott Katzberger, Susan Hoss, Rick Lichner, and Jeff Dutcher for diligently collecting fecal samples for a 15 month time period and providing insight into the daily lives of their animals. Also, many thanks to Drs W Wyatt Hoback, Janet Steele, and Joseph Springer at the Department of Biology, University of Nebraska, for their support and input.

\section{Conflict of interest}

The authors declare no financial interest or any conflict of interest for the purpose of this work.

\section{References}

1. Owen MA, Swaisgood RR, Czekala NM, et al. Monitoring stress in captive giant pandas (Ailuropoda melanoleuca): Behavioral and hormonal response to ambient noise. Zoo Biology. 2014;23(2):147-164.

2. Carlstead, K, Brown JL. Relationships between patterns of fecal corticoid excretion and behavior, reproduction, and environmental factors in 
captive black (Diceros bicornis) and white (Ceratotherium simum) rhinoceros. Zoo Biology. 2005;(24):215-232.

3. Birke L. Effects of browse, human visitors, and noise on the behaviour of captive orangutans. Animal Welfare. 2002;11(2):189-202.

4. Carlstead K, Mellen J, Kleiman DG. Black rhinoceros (Diceros bicornis) in U.S. zoos: I. Individual behavior profiles and their relationship to breeding success. Zoo Biology. 1999;(18):17-34.

5. Carlstead K, Fraser J, Bennett C, et al. Black rhinoceros (Diceros bicornis) in U.S. zoos: II. Behavior, breeding success, and mortality in relation to housing facilities. Zoo Biology. 1999;(18):35-52.

6. Wielebnowski NC. Stress and distress: evaluating their impact for the well-being of zoo animals. J Am Vet Med Assoc. 2003;223(7):973-977.

7. Mostl E, Palme R. Hormones as indicators of stress. Domest Anim Endocrinol. 2002;23(2):67-74.

8. Millspaugh JJ, Washburn BE. Use of fecal glucocorticoid metabolite measures in conservation biology research: considerations for application and interpretation. Gen Comp Endocrinol. 2004;138(3):189-199.

9. Morgan KN, Tromborg CT. Sources of stress in captivity. Applied Animal Behavior Science. 2008;102:262-302.

10. Anthony A, Ackerman E, Lloyd JA. Noise stress in laboratory rodents: I. Behavioral and endocrine responses of mice, rats, and guinea pigs. Journal of the Acoustical Society of America. 1959;31(11):1437-1440.

11. Gamble MR. Sound and its significance for laboratory animals. Biological Reviews of the Cambridge Philosophical Society. 2002;57:395-421.

12. Patterson Kane EG, Farnworth MJ. Noise exposure, music, and animals in the laboratory: A commentary based on laboratory animal refinement and enrichment forum (LAREF) discussions. J Appl Anim Welf Sci. 2006;9(4):327-332.

13. Sato $H$, Takigawa $H$, Sakamoto $H$. Noise effects of reproduction function in rats (author's translation). Nippon Eiseigaku Zasshi. 1982;(36):833843

14. Turner JW, Tolson P, Hamad N. Remote assessment of stress in white rhinoceros (Ceratotherium simum) and black rhinoceros (Diceros bicornis) by measurement of adrenal steroids in feces. J Zoo Wildl Med. 2002;33(3):214-221

15. Teixeira CP, Schentini de Azevedo C, Mendl M, et al. Revisiting translocation and reintroduction programmes: The importance of considering stress. Animal Behavior. 2007;(73):1-13.

16. Cody RP, Smith JK. Applied statistics and the SAS programming language. 4th ed. USA: Prentice Hall;1977.

17. Wingfield JC. The concept of allostasis: Coping with a capricious environment. Journal of Mammology. 2005;86(2):248-254.

18. Hosey GR. Zoo animals and their human audience: What is the visitor effect. Animal Welfare. 2000;9:343-358.

19. Shepherdson DJ, Carlstead KC, Wielebnowski NC. Cross-institutional assessment of stress response in zoo animals using longitudinal monitoring of faecal corticoids and behavior. Animal Welfare. 2004;13:105-113.
20. Wielebnowski NC, Fletchall N, Carlstead K, et al. Noninvasive assessment of adrenal activity associated with husbandry and behavioral factors in the North American clouded leopard population. Zoo Biology. 2002;21:77-98.

21. Montanha JC, Silva SL, Boere V. Comparison of salivary cortisol concentrations in jaguars kept in captivity with differences in exposure to the public. Santa Maria. 2009;39(6):1745-1751.

22. Mitchell G, Tromborg CT, Kaufman J, et al. More on the "influence" of zoo visitors on the behaviour of captive primates. Applied Animal Behavior Science. 1992;35:189-198.

23. Lambeth SP, Bloomsmith MA, Alford PL. Effects of human activity on chimpanzee wounding. Zoo Biology. 1997;16:327-333.

24. Hosey GR. How does the zoo environment affect the behaviour of captive primates. Applied Animal Behavior Science. 2005;(90):107-109.

25. Kyngdon DJ, Minot EO, Stafford KJ. Behavioural responses of captive common dolphins (Delphinus delphis) to a "swim-with-dolphin" programme. Applied Animal Behavior Science. 2003;(81):163-170.

26. Nogueira SSC, Bernadi LG, Nogueira SLG. A note on comparative enclosure facility usage by wild and captive-born capybaras (Hydrochoerus hydrochaeris). Applied Animal Behavior Science. 2004;(89):139-143.

27. Jildge B. The rabbit a diurnal or nocturnal animal. J Exp Anim Sci. 1991;34(5):170-183.

28. Weisenberger ME, Krausman PR, Wallace MC, et al. Effects of simulated jet aircraft noises on hear rate and behavior of desert ungulates. Journal of Wildlife Management. 1996;(60):52-61.

29. Von Muggenthaler E. Infrasound from the Rhinocerotidae. In: Proceedings of the International Conference of Rhinoceros Biology and Conservation, 1992. p. 136-140.

30. Von Muggenthaler E. Low frequency and infrasonic vocalizations from tigers. The journal of Acoustical Society of America. 2000;108(5).

31. Krebs H, Weyers $\mathrm{P}$, Macht $\mathrm{P}$, et al. Scanning behavior of rats eating under stressful noise. Physiol Behav. 1997;62(1):151-154.

32. Maier JAK, Murphy SM, White RG, et al. Responses of caribou to over flights by low-altitude jet aircraft. Journal of Wildlife Management. 1998;62(2):752-766.

33. Weiss JM. Effects of coping responses on stress. Journal of Comparative Physiology and Psychology. 1968;65(2):251-260.

34. Ursin H, Olff M. Psychobiology of coping and defense strategies. Neuropsychobiology. 1993;28(2), 66-71.

35. Henry JP, Stephens PM. Health and the social environment: A sociobiological approach to medicine. Germany: Springer;1997.

36. Watson R, Munro C, Edwards KL, et al. Development of a versatile enzyme immunoassay for non-invasive assessment of glucocorticoid metabolites in a diversity of taxonomic species. Gen Comp Endocrinol. $2013 ; 186: 16-24$ 